FASD fill our jails.' There are 25 differential diagnoses to FASD. Many with FASD are misdiagnosed as Autism Spectrum Disorder. In the single UK clinic for FASD assessment, 49\% of attendees were pre-diagnosed with ASD. There are trans generational aspects concerning the cycle of addiction from generation to generation. Those with FASD are at increased risk of addiction to alcohol. Prevention of FASD can break this cycle.

In Ireland the evidence indicates that: four in five of first pregnancies are exposed to alcohol; nearly one in two (45\%) are exposed at high-risk levels. Two in five pregnancies are unplanned, increasing the chance they will be exposed to alcohol. Pregnant women do not consistently receive timely maternity care or support for their Alcohol \& Drug issues. Health professionals do not consistently provide information on the risks of drinking during pregnancy or routinely screen for alcohol issues. Most clinicians lack the capability to diagnose FASD. Families of people with FASD struggle to access appropriate support and report a lack of understanding from services, professionals and even other family members. FASD affects children and young people in child care and protection services.

The elephant in the room: to what extent is ASD a manifestation of FASD? FASD diagnosis requires documented prenatal alcohol exposure - this limits ascertainment. Universal screening \& brief intervention for alcohol; and at-risk group Parent Child Assistance Programmes, are effective public health interventions in the prevention of FASD.

Knowledge is power.Those with FASD deserve the correct diagnosis towards understanding and acceptance. Paediatricians are key to this diagnosis. Active surveillance for FAS in at risk populations is undertaken in other jurisdictions. Namely, children in state care, young offenders, the prison population; and population based surveillance within national schools, whereby all those less than 10th/25th centile for growth (height) are screened for FAS. While FAS is suited to screening, the diagnosis of FASD is more challenging. Both are underdiagnosed and under ascertained. Prevalence data is needed to make the business case for public health interventions.

\section{P137 AN AUDIT TO CHECK THE AWARENESS AMONG PAEDIATRIC NCHD ABOUT DOCUMENTATION OF CHILDHOOD DEVELOPMENT IN CLINIC LETTERS IN WEXFORD GENERAL HOSPITAL}

Muhammad Zia*, Naeem Shori, Muhammad Jawad, Muhammad Azam. Wexford General hospital, wexford, Ireland

\subsection{6/archdischild-2019-epa.492}

Introduction Development is the period of physical, cognitive and social growth that begins at birth and continues through childhood. Between 1-3\% of children suffer from global delay. Maximum development occurs in the first two of years of life .Early recognition of developmental issues is important to have a better outcome, appropriate referral and involvement of other services.

Aim Aims of the audit is to see the awareness among NCHD (non consultant hospital doctors) towards development assessment and documentation in clinical letters of patients coming to clinic from Jan 2018 to July 2018.
Methodology Retrospective audit was done from January 2018 to July 2018.

A Proforma was designed and random clinic letters of patients attending paediatric clinic were screened for documentation of all six areas of childhood development (gross motor, fine motor vision ,hearing ,speech and language and social) seen by NCHD.

Results

1. Total 70 patients were included in the audit.

2. 45 male and 55 female patients.

3. Age range varies from 3 months to 13 years.

4. $35(50 \%)$ of the patients were below 5 years of age.

5. Childhood development was mentioned in 30(43\%) of patients.

6. $40(57 \%)$ patients have no records of being screened for appropriate age related developmental milestones.

7. Out of 70 only 6 patients (8\%) have their proper documentation about the development indicating all six areas of development.

8. Regarding different areas of development documentation was as follows:

Gross motor 14(20\%) Fine motor 10(14\%) Vision 07(10\%) Speech 12(17\%) Hearing 06(08\%) Social 11(15\%)

Conclusion Developmental assessment is a neglected area in general paediatrics clinic settings.

Outcome about the documentation of general paediatric development assessment was very poor as $57 \%$ of the patients have no records of any development assessment in clinic.

Recommendations

1. Awareness should be raised among NCHDs about the importance of developmental assessment in children coming to clinic.

2. A regular training session for new doctors about routine developmental screen at the start of rotation may be helpful.

3. There should be a brief developmental check list and clinic letter layout displayed in clinic area settings.

4. More opportunities should be given to NCHDs to attend developmental clinics

\section{P138 IMPACT OF CHRONIC PAIN ON ADOLESCENTS' SCHOOL FUNCTIONING: A SYSTEMATIC REVIEW} 1,2Fatimah Alsaggaf*, ${ }^{1}$ Imelda Coyne. ${ }^{1}$ Trinity College of Dublin, Dublin, Ireland; ${ }^{2}$ King
Abdul-Aziz University, Jeddah, Saudi Arabia

\subsection{6/archdischild-2019-epa.493}

Background Chronic pain that persists for more than three months and continues beyond the normal period of tissue healing is considered as a significant health problem among children. Chronic pain has a significant impact on children and adolescents' daily activities. Importantly, school functioning including school attendance, academic achievement, perceptions of self and teacher towards academic competence, classroom attention, participation in school activities, and social functioning, appears to be influenced by paediatric chronic pain. It is therefore important to manage the pain of children and adolescents in the school settings in order to minimise impact on school functioning.

Objective This aim of this review was to critically synthesise current empirical studies on the impact of chronic pain on 
adolescents' school functioning and the pain management interventions in school settings for adolescents with chronic pain.

Methods A systematic review was conducted on school functioning in adolescents aged 13-18 years with chronic pain and pain management in the school setting. A comprehensive search of seven databases; Medline, CINAHL, PsycINFO, ERIC, ScienLO, Cochrane Library, and EMBASE databases, was accomplished covering the period from January $1^{\text {st }}, 1976$ to December $31^{\text {st }}, 2018$.

Results The studies on the topic of school functioning reported that chronic pain has a significant influence on school attendance. Adolescents with chronic pain appear to miss more days than healthy peers. In addition, chronic pain among adolescents is associated with a sense of isolation. This population see themselves as different than their school classmates and peers. However, other studies indicate that chronic pain has no effect on adolescents' academic competence. In order to overcome the struggles of adolescents with chronic pain face in the school settings, studies suggested that the medical team and family members should work in tandem with the teachers to enable an appropriate response to children's chronic pain.

\section{P139 PARTIAL TRISOMY 3P - A CASE REPORT}

Niofa Canty*, Aoife Branagan, Evanne O'Halloran, Michael B O'Neill, Hilary Stokes. Department of Paediatrics, Mayo University Hospital, Castlebar, Co Mayo, Ireland

\subsection{6/archdischild-2019-epa.494}

Introduction Partial trisomy $3 \mathrm{p}$ results from an unbalanced translocation or de novo duplication. Clinical features include of structural brain abnormalities, dysmorphic facies, congenital heart defects, learning disability and hypoplastic genitalia. We report the clinical manifestations of a case of partial trisomy $3 \mathrm{p}$.

Case report The female proband, now 3 years and 9 month female was born to a 33 year old Caucasian mother with 2 neurodevelopmentally normal male children. Intrauterine growth restriction and microcephaly identified antenatally prompted investigation. Fetal MRI at 21 weeks gestation demonstrated dilation of the occipital horn of the right lateral ventricle. Amniocentesis confirmed partial trisomy of chromosome 3

The infant was born at term with no resuscitation or respiratory support required. Echocardiogram revealed a secundum atrial septal defect, muscular ventricular septal defect and a restrictive patent ductus arteriosus. Neonatal MRI demonstrated several structural abnormalities:

1. A parieto-occipital encephalocele measuring $2 \times 3.7 \mathrm{~cm}$ containing brain tissue and cerebrospinal fluid

2. Dilation of the occipital horn

3. Bilateral atresia of lateral ventricles

4. Areas of cortical malformation

5. An abnormal corpus callosum

Abdominal and hip ultrasound examination were normal.

The encephalocele was successfully repaired in the neonatal period. Post operative recovery was complicated by development of hydrocephalus requiring a ventriculo-peritoneal shunt. At 10 and 18 months the patient had seizures related to intercurrent illness and fever. She has never had an afebrile seizure.

At present this child is 3 years 9 months with global developmentally delay in all domains. She can walk unaided but has difficulty running. She can use stairs with support but has not progressed to cycling a tricycle. She is visually impaired secondary to a cortical impairment, requiring corrective lenses. She has bilateral sensorineural hearing loss with a cochlear implant. E has remained medically well to this point and has avoided hospital admissions. She requires intensive multidisciplinary involvement by community allied health professionals including physiotherapy, speech and language therapy and occupational therapy. Her parents hope she will be able to access mainstream education with support.

Given the rarity of her condition with few reported cases in the medical literature, her long term developmental outcome remains unclear.

\section{REFERENCES}

1. Partial $3 p$ trisomy and different rearrangements involving chromosome 3 in the proposita's family, De Pinto Neto et al, American Journal of Medical Genetics, 1980

\section{P140 8P 23 DELETION: A CASE REPORT ON AN INFANT WITH HYPOTONIA}

Muhammad Zia*, Raef Abouelhussein, Conn Sreenan, Niazy Alasaaf, Rizwan Khan. University Maternity Hospital Limerick, Limerick, Ireland

\subsection{6/archdischild-2019-epa.495}

Background An infant with hypotonia and dysmorphic features.

Case report A pre term baby boy born to Irish traveller parents at 32 weeks gestation. Mom was Gravida 5 Para 4 with history of oligohydramnios during pregnancy.Baby was born by Emergency LSCS because of premature rupture of membranes and absent liquor with birth weight of $2.19 \mathrm{~kg}$. Baby required intubation and surfactant at admission for RDS. Baby was extubated next day.On examination baby was noticed to have depressed nasal bridge, hypertelorism, high forehead,bilateral reducible inguinal hernias,posterior plagiocephaly with hypotonia.A normal male genitalia with both testis in scrotum identified. Hypotonia persisted with poor feeding requiring NG feeds.X rays showed thoracic scoliosis with curvature convex to right along with hemivertebrae T4-T9. Infant was also having left sided hydronephrosis with hydroureter and normal right kidney.He had normal newborn hearing screen.Ophthalmologic examination showed underdeveloped macula and hypoplasia of fovea.MRI examination showed evidence of Agenesis of corpus collasum.In view of multiple congenital anomalies microarray was requested that confirmed the diagnosis of $8 \mathrm{p} 23$ deletion. There was no famliy history of note.Currently baby is 14 weeks old and still requiring nasograstic feeds.

Discussion 8p 23 deletion is a rare syndrome that was first described in 1976 by Weleber et.al.It has an estimated prevalence rate of about 1:20000 newborns.Patients display moderate to severe developmental delay,characteristic facial features, congenital heart defects,hypotonia,skeletal abnormalities such as scoliosis and agenesis of the corpus collasum. Prognosis is mainly dependant upon the severity of the signs and symptoms and its associated complications. 